\title{
Practical usage of multiple-choice questions as part of learning and self-evaluation
}

Paula Kangasniemi 2.-3.6.2016, Lapland University Consortium Library

Paula Kangasniemi 2.-3.6.2016, Lapland University Consortium Library

Which one of these arguments is true?

A subject term search is performed on fields describing the contents of the publication.

A subject term search is performed on all fields.

Each publication has only one correct subject term. A subject term search usually gives you more search
results than a keyword search.

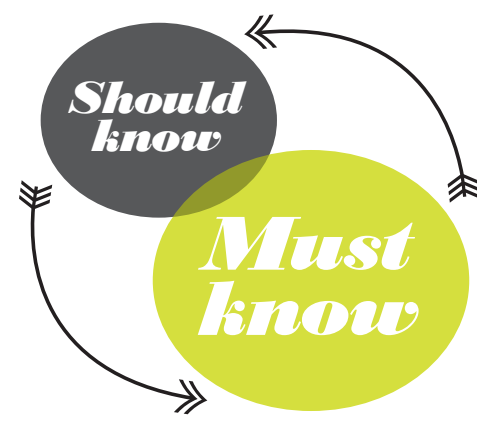

The most important addition to the questions is feedback during practice.

The question's answers (wrong or right) are not important. The feedback on the answers are important to guide students how to do searching. The questions promote the selfregulation and self-evaluation of students.
Feedback can be verbal, screenshot or a video.

\section{Web of Science}

You should find articles about leadership AND job satisfaction. Limit the search to "topic"

and put quotation marks around the phrase "job satisfaction".

Refine your search results to Social Sciences (on the left)

NOTE! You can check the availability of the article by clicking SFX-linking.

Which of the following statements are correct? NOTE! You can choose one or more statements.

X There are over 2400 references about my topic. Feedback: Did you forget the quotation marks? If you did, the system is

How can multiple choice questions promote learning?

What do you have to take into account, when you are preparing multiple-choice questions as mediators?

First, you can prioritize teaching objectives:

-What must be known and what should be known?

-What kind of searches you want to urge students to try?

According to your experiences with contact learning, you can assess,

what the students have already practiced. What are the things

that students have problems with and need more guidance on? performing the search on job, leadership and satisfaction. It is much broader than "job satisfaction".

\ "It's all a matter of consensus" (2014) is available as full text. Feedback: By clicking the SFX symbol you can find out that the article is available in SAGE.

$\bigotimes$ You can refine your search with suggested research areas or Web of Science categories. Feedback: There are various ways of refining the results available on the left column.

\ "Pulling the Levers: Transformational Leadership, Public Service Motivation, and Mission Valence" is published in Public Administration Review, vol 72, issue 2, 2012. Feedback: You can refine your results also by searching some words. Look at a key screenshot! -

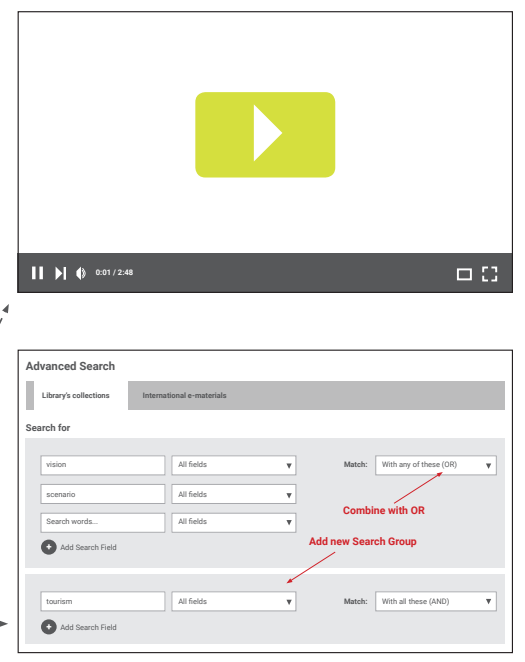

We need new ways of thinking about learning and how we create learning spaces. The key design choices for creating a positive learning experience involve feedback, flexibility, functionality, fun, and freedom. 